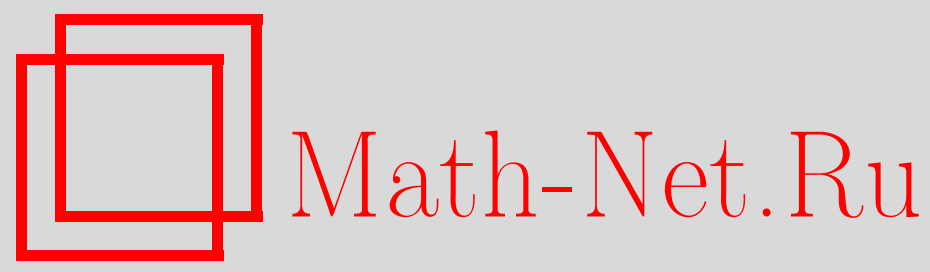

В. И. Парусников, Обобщение теоремы Пинкерле для $k$ членных рекуррентных соотношений, Матем. заметки, 2005, том 78, выпуск 6, 892-906

DOI: https://doi.org/10.4213/mzm2661

Использование Общероссийского математического портала Math-Net.Ru подразумевает, что вы прочитали и согласны с пользовательским соглашением http://www.mathnet.ru/rus/agreement

Параметры загрузки:

IP : 3.89.197.203

26 апреля 2023 г., $17: 50: 34$ 


\section{ОБОБЩЕНИЕ ТЕОРЕМЫ ПИНКЕРЛЕ ДЛЯ $k$-ЧЛЕННЫХ РЕКУРРЕНТНЫХ СООТНОШЕНИЙ}

\section{В. И. Парусников}

В 1894 г. Пинкерле доказал теорему, связывающую существование минимального решения трехчленных рекуррентных соотношений со сходимостью определенной цепной дроби. В настоящей работе рассматриваются решения бесконечной системы $k$-членных рекуррентных соотношений

$$
q_{n}=\sum_{j=1}^{k-1} p_{k-j, n} q_{n-j}, \quad p_{1, n} \neq 0, \quad n=0,1, \ldots,
$$

с коэффициентами из некоторого поля $F$. Изучается связь таких соотношений и многомерных $((k-2)$-мерных) цепных дробей. Устанавливается многомерный аналог теоремы Пинкерле.

Библиографоия: 13 названий.

1. Введение. При вычислении таких решений бесконечной системы $(m+2)$-членных $(m \in \mathbb{N})$ рекуррентных соотношений

$$
q_{n}=\sum_{j=1}^{m+1} p_{m+2-j, n} q_{n-j}, \quad p_{1, n} \neq 0, \quad n=0,1, \ldots,
$$

которые малы относительно других решений, исследователи сталкиваются с тем обстоятельством, что малая абсолютная погрешность в начальных условиях (т.е. добавка другого решения) при вычислениях по рекуррентной формуле сохраняется, но приводит к росту относительной ошибки. Для $m=1$ это имеет место, например, для функций Бесселя первого рода [1]. В 1894 г. Пинкерле доказал (см. [2], а также [3]) следующее утверждение.

ТЕорема (Пинкерле). А) Цепная дробь

$$
c_{0}+\frac{d_{1}}{c_{1}+\frac{d_{2}}{\ddots}}
$$

Работа выполнена при финансовой поддержке Российского фонда фундаментальных исследований, гранты № 03-01-00027, № 02-01-01067 и № 05-01-00050, программы “Современные проблемы теоретической математики" ОМН РАН. 
сходится в $\mathbb{R} \cup\{\infty\}$ тогда и только тогда, когда у системы трехчленных рекуррентных соотношений

$$
y_{n+1}=c_{n} y_{n}+d_{n} y_{n-1}, \quad n=1,2, \ldots
$$

есть минимальное решение $\left\{h_{n}\right\}, h_{n} \in \mathbb{R}$.

Б) В случае сходимости иепной дроби минимальное решение может быть представлено как

$$
-\frac{h_{n}}{h_{n-1}}=\frac{d_{n}}{c_{n}+\frac{d_{n+1}}{c_{n+1}+\frac{d_{n+2}}{\ddots}}}, \quad n=1,2, \ldots
$$

(Решение $\left\{h_{n}\right\}$ системы (3) минимальное, если для непропорциональных ему решений $\left\{g_{n}\right\}$ имеет место $\lim _{n \rightarrow \infty} h_{n} / g_{n}=0$.)

В настоящей работе для некоторого класса полей $F$ изложена теория многомерных цепных дробей. Изучаются $(m+2)$-членные рекуррентные соотношения с коэффициентами из $F$ и их связь $c m$-мерными цепными дробями. Для многомерных цепных дробей доказьвается теорема, обобщающая на многомерньй случай теорему Пинкерле. Представленные здесь материалы частично приведены в препринте [4].

2. Основные определения. Пусть имеется некотороеполе $K$, снабженное топологией, относительно которой непрерьвны операции сложения и умножения. Далее, пусть $K$ непрерывно вложено в полное топологическоепространство $F$, в котором оно плотно. При этом $F$ - также поле. Примерами таких полей $K$ будут поля алгебраических чисел (поле $F=\mathbb{R}$ или $F=\mathbb{C}$ ), поле $R_{p} p$-адических чисел, поле рациональных функций $\widetilde{F}(X)$ над другим полем $\widetilde{F}$, поле $\widetilde{F}((X))$ формальных степенных рядов

$$
f=\sum_{r=n}^{\infty} c_{r} X^{r}, \quad c_{r} \in \widetilde{F}, \quad n \in \mathbb{Z},
$$

над $\widetilde{F}$.

Через $F^{m}, m \in \mathbb{N}$, обозначим $m$-мерноевекторное пространство надполем $F, F^{m} \stackrel{\text { def }}{=}$ $F^{m} \backslash(0, \ldots, 0)$, а через $F \mathbb{P}^{m}, m \in \mathbb{Z}^{+}$, обозначим $m$-мерное проективное простран$c m в о$, т.е. множество классов эквивалентности $F^{m+1^{\prime}}$ по отношению пропорциональности ' $\sim$ ':

$$
\left(\mathbf{f}_{1} \sim \mathbf{f}_{2}, \mathbf{f}_{1}, \mathbf{f}_{2} \in F^{m+1^{\prime}}\right) \Longleftrightarrow\left(\exists f_{12}, f_{21} \in F^{\prime}, f_{12} \mathbf{f}_{1}=f_{21} \mathbf{f}_{2}\right)
$$

Однородные координаты проективных векторов будем нумеровать от 1 до $m+1$, между ними будем ставить двоеточие: $\left(f_{1}: \cdots: f_{m+1}\right)$. Векторы и проективные векторы (элементы $F^{m}$ и $F \mathbb{P}^{m}$ ) будем писать жирньп шрифтом, их компоненты располагать в виде вектор-строк, но в матричных операциях считать вектор-столбцами. Проективный вектор с единственной ненулевой $k$-й компонентой обозначим через $\mathbf{e}_{k}$, а вектор $(0, \ldots, 0) \in F^{m}$ - через $\mathbf{0}$. Элемент $i$-й строки и $j$-го столбца $(k \times k)$-матрицы $M$ обозначим $(M)_{i, j}$, а $i$-ю компоненту вектора $\mathbf{f}$ - через $(\mathbf{f})_{i}$. Подобное обозначение для однородных координат проективных векторов $\mathbf{f} \in F \mathbb{P}^{m}$ будет использоваться в условиях 
типа $(\mathbf{f})_{j}=0$ или $(\mathbf{f})_{j} \neq 0$. Единичную матрицу обозначим через $E$, символ Кронеккера - через $\delta_{i, j}, \mathbb{Z}^{+}=\mathbb{N} \cup\{0\}$.

Отождествим множество

$$
F \mathbb{P}_{m+1}^{m} \stackrel{\text { def }}{=}\left\{\mathbf{f} \in F \mathbb{P}^{m} \mid(\mathbf{f})_{m+1} \neq 0\right\} \subset F \mathbb{P}^{m}
$$

с пространством $F^{m}$ при помощи вложения $\sigma: F^{m} \rightarrow F \mathbb{P}_{m+1}^{m}$ :

$$
\begin{gathered}
\sigma\left(f_{1}, \ldots, f_{m}\right) \stackrel{\text { def }}{=}\left(f_{1}: \cdots: f_{m}: 1\right) \\
\sigma^{-1}\left(f_{1}: \cdots: f_{m+1}\right)=\left(\frac{f_{1}}{f_{m+1}}, \ldots, \frac{f_{m}}{f_{m+1}}\right) ; \quad \sigma(\mathbf{0})=\mathbf{e}_{m+1} .
\end{gathered}
$$

В $F \mathbb{P}^{m}$ действует группа дробнолинейных преобразований Aut $F \mathbb{P}^{m}$. Ее элементами служат классы эквивалентности (с точностью до умножения на скаляры из $F^{\prime}$ ), представители которых суть невырожденные матрицы $D$ размера $m+1$ с элементами $d_{i, j}$ :

$$
D\left(f_{1}: \cdots: f_{m+1}\right)=\left(\sum_{j=0}^{m} d_{0, j} f_{j}: \cdots: \sum_{j=0}^{m} d_{m, j} f_{j}\right) .
$$

Матрицы

$$
\left(\begin{array}{cccc}
d_{1,1} & \ldots & d_{1, m} & 0 \\
\ldots & \ldots & \ldots & \ldots \\
d_{m, 1} & \ldots & d_{m, m} & 0 \\
0 & \ldots & 0 & 1
\end{array}\right), \quad\left(\begin{array}{cccc}
1 & \ldots & 0 & 0 \\
\ldots \ldots & \ldots & \ldots \\
0 & \ldots & 1 & 0 \\
0 & \ldots & 0 & d^{-1}
\end{array}\right), \quad\left(\begin{array}{cccc}
1 & \ldots & 0 & d_{1} \\
\ldots & \ldots & \ldots & \ldots \\
0 & \ldots & 1 & d_{m} \\
0 & \ldots & 0 & 1
\end{array}\right)
$$

отвечают следуюшим преобразованиям пространства $F^{m}$ : линейному преобразованию с матрицей $d_{i, j}$, умножению на скаляр $d \neq 0$ и параллельному переносу на вектор $\left(d_{1}, \ldots, d_{m}\right) \in F^{m}$. Матрицу - представитель класса $\left\{c D \mid c \in F^{\prime}\right\}$ - будем выбирать наиболее простой по виду.

3. Операция обращения вектора и цепные дроби. Определим поворот $\mathscr{J}$ пространства $F \mathbb{P}^{m}$ формулой

$$
\mathscr{J}\left(f_{1}: \cdots: f_{m+1}\right)=\left(f_{m+1}: f_{1}: \cdots: f_{m}\right)
$$

$(m+1)$-я степень преобразования $\mathscr{J}$ - это тождественное преобразование, поэтому $\mathscr{J}^{-1}=\mathscr{J}^{m}$ и $\mathscr{J}^{-1}\left(f_{1}: \cdots: f_{m+1}\right)=\left(f_{2}: \cdots: f_{m+1}: f_{1}\right)$. Преобразования $\mathscr{J}, \mathscr{J}^{-1}$ имеют соответственно матрицы

$$
\mathscr{J}=\left(\begin{array}{cccc}
0 & \ldots & 0 & 1 \\
1 & \ldots & 0 & 0 \\
\ldots & \ldots & \ldots & \\
0 & \ldots & 1 & 0
\end{array}\right), \quad \mathscr{J}^{-1}=\left(\begin{array}{cccc}
0 & 1 & \ldots & 0 \\
\ldots \ldots \ldots & \ldots & \\
0 & 0 & \ldots & 1 \\
1 & 0 & \ldots & 0
\end{array}\right)
$$

С помощью этих преобразований на “почти всем" пространстве $F^{m}$ можноввести две взаимно обратные операции обращения векторов из $F^{m}$. Для прослеживания параллели с теорией одномерных цепных дробей одну операцию будем записьвать с помощью одной горизонтальной черты, а другую - с помощью двух черточек. Итак,

$$
\frac{1}{\mathbf{f}} \stackrel{\text { def }}{=} \sigma^{-1} \mathscr{J} \sigma \mathbf{f}, \quad \frac{1}{\overline{\mathbf{g}}} \stackrel{\text { def }}{=} \sigma^{-1} \mathscr{J}^{-1} \sigma \mathbf{g}, \quad \mathbf{f}, \mathbf{g} \in F^{m}, \quad(\mathbf{f})_{m} \neq 0, \quad(\mathbf{g})_{1} \neq 0 .
$$


В координатах эти формулы выглядят так:

$$
\begin{gathered}
\frac{1}{\left(f_{1}, \ldots, f_{m}\right)}=\left(\frac{1}{f_{m}}, \frac{f_{1}}{f_{m}}, \ldots, \frac{f_{m-1}}{f_{m}}\right), \quad \mathbf{f} \in F^{m}, \quad(\mathbf{f})_{m} \neq 0 \\
\frac{1}{\overline{\left(g_{1}, \ldots, g_{m}\right)}}=\left(\frac{g_{2}}{g_{1}}, \ldots, \frac{g_{m}}{g_{1}}, \frac{1}{g_{1}}\right), \quad \mathbf{g} \in F^{m}, \quad(\mathbf{g})_{1} \neq 0 .
\end{gathered}
$$

Отметим, что

$$
\frac{1}{\left(\frac{1}{\mathbf{f}}\right)}=\mathbf{f}, \quad \frac{1}{\left(\frac{1}{\bar{g}}\right)}=\mathbf{g}, \quad \mathbf{f}, \mathbf{g} \in F^{m}, \quad(\mathbf{f})_{m} \neq 0, \quad(\mathbf{g})_{1} \neq 0 .
$$

Разложение в иепную дробь состоит из ряда однотипных шагов, в свою очередь, состоящих из трех этапов: выделения "целой части", выделения из остатка множителя ("числителя") и обращения остатка. Простейший вариант приведенного ниже алгоритма впервые к числовым векторам применил Якоби [5], а в частньх случаях этот алгоритм рассматривал Эйлер [6]: все числители равнялись 1 и целая часть вектора бралась покомпонентно. Шаг с номером $n \in \mathbb{Z}^{+}{ }_{-}$это получение из вектора $\mathbf{f}_{n}\left(\mathbf{f}_{0}=\mathbf{f}\right)$ вектора $\mathbf{f}_{n+1}$ такого, что

$$
\mathbf{f}_{n}=\mathbf{b}_{n}+\frac{a_{n}}{\mathbf{f}_{n+1}} \quad \text { или } \quad \mathbf{f}_{n+1}=\frac{1}{\overline{\left(\mathbf{f}_{n}-\mathbf{b}_{n}\right) a_{n}^{-1}}} .
$$

(Как обычно, здесь сначала выполняется обращение вектора, затем умножение на скаляр, и в последнюю очередь сложение.) Отметим, что

$$
\frac{1}{\overline{\left(\mathbf{f}_{n}-\mathbf{b}_{n}\right) a_{n}^{-1}}} \neq \frac{a_{n}}{\overline{\mathbf{f}_{n}-\mathbf{b}_{n}}}
$$

при $m>1, a \neq 1$.

На первом этапе алгоритма отыскивается $n$-й знаменатель $\mathbf{b}_{n}$ иепной $\partial p о б u-$ выделяется "целая часть" $\left[\mathbf{f}_{n}\right]$ вектора. При этом уже для $m=1$ и $F=\mathbb{R}$ “целой частью" может быть и взятие ближайшего целого, и ближайшего четного, и другие более сложные операции. Напрашивающееся в многомерном случае покомпонентное взятие "целой части" $\left(\mathbf{b}_{n}=\left(\left[(\mathbf{f})_{1}\right], \ldots,\left[(\mathbf{f})_{m}\right]\right)\right)$ тоже не единственньй способ определения этой операции. "Целой частью" [· ] там может служить любое относительно "хорошее" в топологическом смысле отображение пространства $F^{m}$ в некоторый модуль $M$ над некоторым содержащимся в $K$ кольцом $R$. Требуется, чтобы это отображение было неподвижно в точках модуля $M$, т.е. было проектором. Удобно также требовать инвариантность этого отображения относительно сдвигов на элементы модуля $M:[\mathbf{x}+\mathbf{y}]=[\mathbf{x}]$ для $\mathbf{x} \in F^{m}, \mathbf{y} \in M$.

Разность $\mathbf{g}_{n} \stackrel{\text { def }}{=} \mathbf{f}_{n}-\mathbf{b}_{n}$ назьвается $n$-ьлм остатком цепной дроби. На втором этапе из остатка выделяют ненулевой множитель $a_{n} \in K^{\prime}-$ числитель иепной дроби. Вместе числители $a_{n}$ и знаменатели $\mathbf{b}_{n}$ суть әлементы иепной дроби.

Третий этап - обращение полученного на предшествующем этапе вектора

$$
\mathbf{h}_{n}=\left(h_{1, n}, \ldots, h_{m, n}\right) \stackrel{\text { def }}{=} \frac{\mathbf{f}_{n}-\mathbf{b}_{n}}{a_{n}} .
$$


Если $g_{1, n}=a_{n} h_{1, n} \neq 0$, то вектор $\mathbf{f}_{n+1}$ находится по формуле (9):

$$
\mathbf{h}_{n}=\frac{1}{\mathbf{f}_{n+1}}, \quad \text { или } \quad \mathbf{f}_{n+1}=\frac{1}{\overline{\mathbf{h}_{n}}}=\left(\frac{h_{2, n}}{h_{1, n}}, \ldots, \frac{h_{m, n}}{h_{1, n}}, \frac{1}{h_{1, n}}\right) .
$$

Если же $g_{1, n}=0$, то обычно говорят об обрыве алгоритма, однако мы назовем обрывом алгоритма (иепной дроби) только случай $\mathbf{g}_{n}=\mathbf{e}_{m+1}$, отличая его от случая $\mathbf{g}_{n} \neq 0$, $g_{1, n}=0$, т.е. надрыва иепной дроби. Ниже мы покажем, как здесь можно продолжить разложение в цепную дробь меньшей размерности. Для $d \in\{0,1, \ldots, m\}$ определим пространства

$$
F^{m, d} \stackrel{\text { def }}{=}\left\{\mathbf{x}=\left(x_{1}, \ldots, x_{m}\right) \in F^{m}: x_{1}=\cdots=x_{d}=0\right\}
$$

Число $d$ такое, что $\mathbf{g}_{n} \in F^{m, d} \backslash F^{m, d+1}$, т.е. $\mathbf{g}_{1, n}=\cdots=\mathbf{g}_{d, n}=0, \mathbf{g}_{d+1, n} \neq 0$, назовем дефектом иепной дроби. Когда дефект цепной дроби стал равным $d>0$, легко предъявить $d$ линейно независимых соотношений

$$
\sum_{j=1}^{m+1} c_{i, j}\left(\mathbf{f}_{0}\right)_{j}, \quad i=1, \ldots, d
$$

связывающих компоненты исходного вектора $\sigma \mathbf{f}_{0}$ и 1 . Линейная зависимость компонент обнаруживается и в случае, когда обращается в нуль не первая, а другая $(k$-я) компонента остатка. Но этот случай выделять не надо: не позже, чем через $k-1$ шаг разложения, первая компонента остатка все же обратится в нуль. Для величин $d$ удобно использовать сдвинутую нумерацию: образовавшийся на $n$-ом шаге дефект будет $d=d(n+1)$.

Некоторые алгоритмы могут выявлять линейную зависимость компонент разлагаемых по ним векторов, т.е. при разложении в цепную дробь таких векторов неизбежно происходит надрыв цепной дроби. Алгоритм в одних полях $F$ может обладать этим свойством, а в других нет. Например, алгоритм Якоби для $F=\mathbb{R}$ не обладает таким свойством при $m \geqslant 3[7]$ и обладает при $m=2$ [8]. Еще пример: в поле $\mathbb{C}((z))$ формальных степенных рядов с полюсом в бесконечности

$$
\mathbb{C}(z)=\left\{f: f=\sum_{k=n}^{\infty} f_{k} z^{-k}, n \in \mathbb{Z}, f_{k} \in \mathbb{C}\right\}
$$

правильные цепные дроби (с числителями 1) алгоритма Якоби при всех $m$ обладают этим свойством [9], а $C$-дроби алгоритма Якоби - нет [10].

Если на шаге $n$ произошел надрьв (но не обрьв) цепной дроби, дальше можно продолжить разложение в цепную дробь меньшей размерности. Изоморфное пространству $F^{m-d}$ подпространство $F^{m, d}$ отождествим с частью проективного пространства

$$
F \mathbb{P}^{m, d} \stackrel{\text { def }}{=}\left\{\mathbf{f} \in F \mathbb{P}^{m} \mid(\mathbf{f})_{1}=\cdots=(\mathbf{f})_{d}=0\right\},
$$

в котором сохраним индуцированную нумерацию координат: $F^{m, d}=\left\{\left(f_{d+1}, \ldots, f_{m}\right) \mid\right.$ $\left.f_{j} \in F\right\}$. Определенный формулой

$$
\mathscr{J}_{d}\left(f_{1}: \cdots: f_{m+1}\right)=\left(f_{1}: \cdots: f_{d}: f_{m+1}: f_{d+1}: \cdots: f_{m-1}: f_{m}\right)
$$


поворот $\mathscr{J}_{d}$ этой части проективного пространства имеет блочную матрицу $\mathscr{J}_{d}$, в левом верхнем углу которой стоит $d$-мерная единичная матрица, а в правом нижнем - по виду такая же, как $\mathscr{J}$, матрища, только размера не $m+1$, а $m+1-d$. Видно, что $\mathscr{J}_{d}^{-1}=\mathscr{J}_{d}^{m-d}, \mathscr{J}=\mathscr{J}_{0}$ и $\operatorname{det} \mathscr{J}_{d}=(-1)^{m-d} \neq 0$.

Описанньй процесс (алгоритм) изображают в виде бесконечной т-мерной иепной $\partial p o б u$

$$
\mathbf{b}_{0}+\frac{a_{0}}{\ddots+\frac{a_{n-1}}{\mathbf{b}_{n}+\frac{a_{n}}{\ddots}}}, \quad a_{n} \in F^{\prime}, \quad \mathbf{b}_{n} \in F^{m} .
$$

Назовем $n$-й подходящей дробъю $\mathbf{u}_{n}$ цепной дроби (11)

$$
\mathbf{u}_{n}=\mathbf{b}_{0}+\frac{a_{0}}{\ddots \mathbf{b}_{n-1}+\frac{a_{n-1}}{\mathbf{b}_{n}}}, \quad n \in \mathbb{Z}^{+} .
$$

Чтобы найти $\mathbf{u}_{n}$, надо “свернуть" агрегат (12) снизу вверх. (Отметим, что при сворачивании мы можем встретиться с ситуацией, когда вектор, полученньй на промежуточном этапе обратить нельзя, так как его последняя компонента обратилась в нуль. Как преодолеть это, мы покажем ниже.) После того, как дано это определение, мы видим, что можно отвлечься от алгоритма разложения, специфики числителей $a_{k}$ и знаменателей $\mathbf{b}_{k}$ цепной дроби и изучать подходящие дроби как самостоятельные объекты с точки зрения их сходимости в том или ином смысле.

Согласно (5) матрицы дробно-линейных преобразований $\mathscr{A}_{n}, \mathscr{B}_{n}$, отвечающих умножению вектора на скаляр $a_{n} \in F^{\prime}$ и сложению с вектором $\mathbf{b}_{n}$, равны

$$
\mathscr{A}_{n}=\left(\begin{array}{cccc}
1 & \ldots & 0 & 0 \\
\ldots & \ldots & \ldots & \ldots \\
0 & \ldots & 1 & 0 \\
0 & \ldots & 0 & a_{n}^{-1}
\end{array}\right), \quad \mathscr{B}_{n}=\left(\begin{array}{cccc}
1 & \ldots & 0 & b_{1, n} \\
\ldots & \ldots & \ldots & \ldots \\
0 & \ldots & 1 & b_{m, n} \\
0 & \ldots & 0 & 1
\end{array}\right) .
$$

Введем $(m+1)$-мерные векторы $\mathbf{p}_{n}=\left(p_{1, n}, \ldots, p_{m+1, n}\right)$ следующим образом:

$$
\mathbf{p}_{n} \stackrel{\text { def }}{=} \begin{cases}\left(a_{n}^{-1}, b_{1, n} a_{n}^{-1}, \ldots, b_{m, n} a_{n}^{-1}\right), & d(n)=0, \\ \left(0, \ldots, 0, a_{n}^{-1}, b_{d(n)+1, n} a_{n}^{-1}, \ldots, b_{m, n} a_{n}^{-1}\right), & d(n)>0,\end{cases}
$$

и матрищы

$$
P_{n} \stackrel{\text { def }}{=} \mathscr{J}_{d(n)} \mathscr{B}_{n} \mathscr{A}_{n}, \quad Q_{-1} \stackrel{\text { def }}{=} E, \quad Q_{n} \stackrel{\text { def }}{=} P_{0} \cdots P_{n}=Q_{n-1} P_{n}, \quad n \in \mathbb{Z}^{+} .
$$

Матрица $P_{n}$ для случаев, когда до $n$-го шага надрыва цепной дроби непроизошло $(d=0)$ и когда цепная дробь имеет дефект $d=d(n)>0$, соответственно равна

$$
P_{n}=\left(\begin{array}{cccc}
0 & \ldots & 0 & p_{1, n} \\
1 & \ldots & 0 & p_{2, n} \\
\ldots & \ldots & \ldots & \ldots \\
0 & \ldots & 1 & p_{m+1, n}
\end{array}\right) \quad \text { или } \quad\left(\begin{array}{ccccc}
E_{d} & \ldots & 0 & \ldots & \ldots \\
\ldots & 0 & \ldots & 0 & p_{d+1, n} \\
\ldots & 1 & \ldots & 0 & p_{d+2, n} \\
0 & \ldots & \ldots & \ldots & \ldots \\
\ldots & 0 & \ldots & 1 & p_{m+1, n}
\end{array}\right)
$$


(Здесь $E_{d}$ - единичная матрица размера $d$, справа и снизу от нее стоят нули.)

Формулы для разлагаемого вектора и подходящих дробей (см. (10), (12)) переписываются как

$$
\begin{aligned}
\mathbf{f}_{0} & =\sigma^{-1} \mathscr{B}_{0} \mathscr{A}_{0} \mathscr{J}_{d(1)} \cdots \mathscr{B}_{n} \mathscr{A}_{n} \mathscr{J}_{d(n+1)} \sigma \mathbf{f}_{n+1}=\sigma^{-1} \mathscr{J}^{-1} Q_{n} \mathscr{J}_{d(n+1)} \sigma \mathbf{f}_{n+1}, \\
\mathbf{u}_{n} & =\sigma^{-1} \mathscr{B}_{0} \mathscr{A}_{0} \mathscr{J}_{d(1)} \cdots \mathscr{B}_{n-1} \mathscr{A}_{n-1} \mathscr{J}_{d(n)} \mathscr{B}_{n} \sigma \mathbf{0}=\sigma^{-1} \mathscr{J}^{-1} Q_{n} \mathbf{e}_{m+1} .
\end{aligned}
$$

(Мы учли, что $\sigma \mathbf{0}=\mathbf{e}_{m+1}$ и что $\mathscr{A}_{n} \mathbf{e}_{m+1}=\mathbf{e}_{m+1}$ для любой $\mathscr{A}_{n}$.)

Рекуррентная формула (13) указывает способ вычисления матрищ $Q_{n}$. Из вида (14) матриц $P_{n}$ получаем, что $m$ из $m+1$ столбцов матрицы $Q_{n-1}$ есть в матрице $Q_{n}$ : левые $d(n)$ столбцов матрищ $Q_{n}$ и $Q_{n-1}$ совпадают, а столбцы $Q_{n}$ с индексами с $(d+1)$-го по $m$-й суть столбцы матрицы $Q_{n-1}$, с индексами на единицу большими. Правый столбец $Q_{n}-$ с индексом $m+1$ - есть линейная комбинация всех, кроме $d$ левых, столбцов матрицы $Q_{n-1}$ :

$$
\left(Q_{n}\right)_{i, m+1}=\sum_{j=d(n)+1}^{m+1} p_{j, n}\left(Q_{n-1}\right)_{i, j} .
$$

Введем для “новых" элементов матриц $Q_{n}$ обозначение $q_{i, n}$. Тогда

$$
\begin{gathered}
q_{i, n}=\delta_{i, n+m+2}, \quad n=-m-1, \ldots,-2,-1, \quad i=1, \ldots, m+1, \\
q_{i, n}=\left(Q_{n}\right)_{i, m+1}, \quad q_{i, n}=\sum_{j=d(n)+1}^{m+1} p_{j, n} q_{i, n-m-2+j}, \quad n \in \mathbb{Z}^{+}, \quad i=1, \ldots, m+1 .
\end{gathered}
$$

Элементы $q_{i, n}$ объединим в одну бесконечную $(m+1)$-строчную матрицу $Q$, столбцы которой будем нумеровать от $-m-1$ до $+\infty$ :

$$
(Q)_{i, n}=q_{i, n}, \quad i=0, \ldots, m, \quad n=-m-1,-m, \ldots, 0,1, \ldots
$$

Формулы (15), (12) и (13) дают следующие выражения для компонент подходящих дробей:

$$
\mathbf{u}_{n}=\left(\frac{q_{2, n}}{q_{1, n}}, \ldots, \frac{q_{m+1, n}}{q_{1, n}}\right) .
$$

Препятствием для существования $\mathbf{u}_{n}$ служит равенство нулю знаменателя $q_{1, n}$. Если же задать $\overline{\mathbf{u}}_{n} \in F \mathbb{P}^{m}$ формулой $\overline{\mathbf{u}}_{n}=\mathscr{J}^{-1} Q_{n} \mathbf{e}_{m+1}$ и не делать переход к $\mathbf{u}_{n}=\sigma^{-1} \overline{\mathbf{u}}_{n}$, то величины $\overline{\mathbf{u}}_{n}$ будут определены всег да. Их компоненты суть $m$ числителей и знаменатель $q_{1, n}$ подходящих дробей. Матрица $\mathscr{J}^{-1} Q_{n}$ невырождена, поэтому $\overline{\mathbf{u}}_{n}=\left(q_{2, n}, \ldots\right.$, $\left.q_{m+1, n}, q_{1, n}\right) \neq \mathbf{0}$.

Если цепная дробь не имеет надрьва, т.е. при $d(n)=0$, члены последовательностей $\mathbf{q}_{i} \stackrel{\text { def }}{=}\left\{q_{i, n}\right\}_{n=-m-1}^{\infty}$ удовлетворяют системе $(m+2)$-членных рекуррентных соотношений

$$
\begin{aligned}
\mathscr{R}\left(\left\{\mathbf{p}_{n}\right\}_{n=0}^{\infty}\right) \stackrel{\text { def }}{=} & \left\{q_{i, n}=\sum_{j=1}^{m+1} p_{m+2-j, n} q_{i, n-j}\right. \\
& \left.\mathbf{p}_{n}=\left(p_{1, n}, \ldots, p_{m+1, n}\right) \in F_{1}^{m+1}\left(p_{1, n} \neq 0\right)\right\}_{n=0}^{\infty}
\end{aligned}
$$

с начальньми условиями (16).

Сформулируем доказанноев виде следующих вариантов известных свойств [8, $1-4]$. 
ТЕоремА 1. Числители $q_{i, n}, i=2, \ldots, m+1, u$ знаменатели $q_{1, n}$ для $n-x$ подходящих дробей

$$
\mathbf{u}_{n}=\left(\frac{q_{2, n}}{q_{1, n}}, \ldots, \frac{q_{m+1, n}}{q_{1, n}}\right)
$$

удовлетворяют рекуррентным соотношениям (18) с начальными условиями (16).

ТЕорема 2. Имеется взаимно однозначное соответствие между

(i) не имеющими надрыва т-мерны.ми иепны.ми дробями (11);

(ii) последовательностями $\left\{\mathbf{p}_{n}\right\}_{n=0}^{\infty}$ векторов $\mathbf{p}_{n} \in F^{m+1},\left(\mathbf{p}_{n}\right)_{1} \neq 0$;

(iii) системами $(m+2)$-членных рекуррентных соотношений (18).

Заметим, что вся изложенная вьше теория верна, хотя и совершенно тривиальна, для $m=0$. У 0-мерной цепной дроби есть только числители $\left\{a_{n}\right\}_{n=0}^{\infty}, a_{n} \neq 0$, а знаменателей нет. У подходящих дробей к ней, наоборот, есть только знаменатели

$$
q_{1, n}=\prod_{k=0}^{n} a_{k}^{-1}
$$

связанные двучленньми рекуррентными соотношениями $q_{1, n}=a_{n}^{-1} q_{1, n-1}$.

4. Рекуррентные соотношения и подпространства пространства $F^{\infty}$. Нам уже встречались бесконечные последовательности (17) с элементами из $F$. Обозначим через $F^{\infty}$ векторное пространство таких последовательностей

$$
F^{\infty} \stackrel{\text { def }}{=}\left\{\mathbf{s}=\left\{s_{n}\right\}_{n=-m-1}^{\infty}: s_{n} \in F\right\} .
$$

Нулем 0 в $F^{\infty}$ будет последовательность, состоящая из одних нулей: $(\mathbf{0})_{n}=0$. Умножение последовательности на элементы $F$ берется покомпонентно. Определим проекции $\kappa_{r}, r=-1,0,1, \ldots$, пространства $F^{\infty}$ на пространство $F^{m+1}: \kappa_{r}(\mathbf{s}) \stackrel{\text { def }}{=}\left(s_{r-m}, \ldots, s_{r}\right)$. Элементы линейных пространств $F^{\infty}$ будем записывать как вектор-строки.

Зафиксируем систему (18) рекуррентных соотношений $\mathscr{R}=\mathscr{R}\left(\left\{\mathbf{p}_{n}\right\}_{n=0}^{\infty}\right)$. Множество последовательностей $\mathbf{s}$ из $F^{\infty}$ с произвольными начальными әлементами $s_{-m-1}$, $\ldots, s_{-1}$, удовлетворяющих уравнениям (18), образует линейное пространство, которое мы обозначим через $\mathscr{S}(\mathscr{R})$. Поскольку вся последовательность при этом определяется заданием ее начальных членов, пространство $\mathscr{S}(\mathscr{R})$ изоморфно $(m+1)$-мерному пространству $F^{m+1}$. Естественным базисом в нем будет определенная нами ранее система решений $\mathbf{q}_{i}, i=1, \ldots, m+1$, начальные члены которых равны $\left(\mathbf{q}_{i}\right)_{j}=\delta_{i, j+m+2}$, $j=-1, \ldots,-m-1$ (см. (16)). Решение s с произвольными начальньми условиями $\kappa_{-1}(\mathbf{s})=\left(s_{-m-1}^{*}, \ldots, s_{-1}^{*}\right) \in F^{m+1}$ будет линейной комбинацией базисных:

$$
\mathbf{s}=\sum_{j=1}^{m+1} s_{j-m-2}^{*} \mathbf{q}_{j}
$$

Отвечающие системам рекуррентных соотношений, т.е. отвечающие $m$-мерным цепным дробям, $(m+1)$-мерные пространства выделяются среди всех $(m+1)$-мерных подпространств пространства $F^{\infty}$ тем, что для базисов в них все матрицы некоторого типа невырождены. 
Для фиксированного базиса $\left(\mathbf{t}_{1}, \ldots, \mathbf{t}_{m+1}\right)$ определим матрицы $\Delta_{n, j}=\Delta_{n, j}\left(\mathbf{t}_{1}, \ldots\right.$, $\left.\mathbf{t}_{m+1}\right)$ формулами

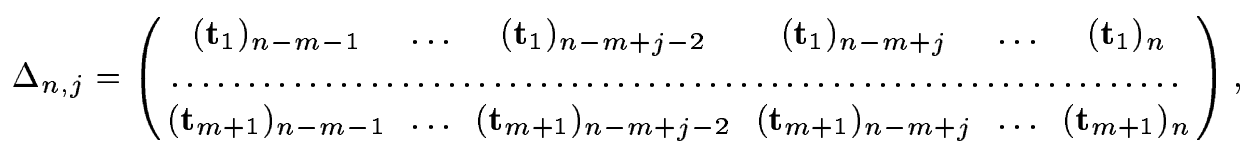

$$
\begin{aligned}
& n \geqslant-1, \quad j=0, \ldots, m+1 \text {. }
\end{aligned}
$$

Невырожденность матриц $\Delta_{n, 0}\left(\mathbf{q}_{1}, \ldots, \mathbf{q}_{m+1}\right)=Q_{n}$ следует из формулы $(13)$ и свойства $\operatorname{det} P_{n}=(-1)^{m} p_{1, n} \neq 0$. Произвольный базис $\mathbf{t}_{1}, \ldots, \mathbf{t}_{m+1}$ пространства $\mathscr{S}(\mathscr{R})$ выражается через базис $\mathbf{q}_{1}, \ldots, \mathbf{q}_{m+1}$ при помощи некоторой невырожденной $(m+1) \times$ $(m+1)$-матрицы $M$ :

$$
\mathbf{t}_{i}=\sum_{j=1}^{m+1}(M)_{i, j} \mathbf{q}_{j}, \quad i=1, \ldots, m+1
$$

Поэтому

$$
\operatorname{det} \Delta_{n, 0}\left(\mathbf{t}_{1}, \ldots, \mathbf{t}_{m+1}\right)=\operatorname{det} M \operatorname{det} Q_{n}=\operatorname{det} M(-1)^{(n+1) m} \prod_{j=0}^{n} p_{1, j} \neq 0 .
$$

Наоборот, пусть теперь матрицы $\Delta_{n, 0}=\Delta_{n, 0}\left(\mathbf{t}_{1}, \ldots, \mathbf{t}_{m+1}\right)$ и $\Delta_{n-1,0}=\Delta_{n-1,0}\left(\mathbf{t}_{1}\right.$, $\ldots, \mathbf{t}_{m+1}$ ) невырож дены. (Это означает, что столбцы этих матриц как векторы $(m+1)$ мерных пространств находятся в общем положсении.) Тогда последний столбец $(m+1) \times(m+2)$-матрицы $\left\{\left(\mathbf{t}_{i}\right)_{j}\right\}_{i=1, \ldots, m+1}^{j=n-m-1, \ldots, n}$ выражается через остальные:

$$
\left(\mathbf{t}_{i}\right)_{n}=\sum_{j=1}^{m+1} p_{j}\left(\mathbf{t}_{i}\right)_{n+j-m-2}
$$

где $p_{j}=(-1)^{m+1-j} \operatorname{det} \Delta_{n, j-1} / \operatorname{det} \Delta_{n-1,0}$, причем $p_{1}=(-1)^{m} \operatorname{det} \Delta_{n, 0} / \operatorname{det} \Delta_{n-1,0}$ $\neq 0$ и вектор $\mathbf{p}=\left(p_{1}, \ldots, p_{m+1}\right)$ лежит в $F_{1}^{m+1}$. Мы пришли к связывающей компоненты векторов $\mathbf{t}_{n}$ рекуррентной формуле. Видно, что отношения определителей независят от матрицы $M$, т.е. от базиса подпространства. Итак, доказана

ТЕОрема 3. Компоненты последовательностей - элементов конечномерного линейного пространства размерности $m+1$ - удовлетворяют системе

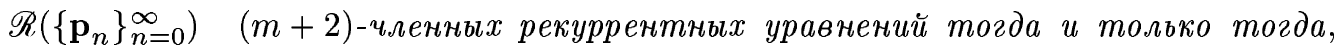
когда для (любого) базиса $\mathbf{t}_{0}, \ldots, \mathbf{t}_{m}$ этого пространства все определители $\Delta_{n}\left(\mathbf{t}_{0}, \ldots, \mathbf{t}_{m}\right), n=-1,0,1,2 \ldots$, отличны от нуля.

Итак, вместо систем рекуррентных соотношений можно рассматривать некоторые конечномерные подпространства пространства $F^{\infty}$ и наоборот.

5. Сходимость цепных дробей и проективных векторов. Рассматривая примеры цепных дробей, мы сталкиваемся с ситуацией, когда подходящие дроби к ним не принадлежат пространству $F^{m}$. В одномерном случае, чтобы избежать постоянных оговорок, связанньх с этим, вместо нормированного поля $F$ рассматривают его пополнение бесконечно удаленным элементом $\{\infty\}: \bar{F}=F \cup\{\infty\}$. Это позволяет наряду с обычной сходимостью цепной дроби (в $F)$ ввести такое понятие сходимости, что изменение цепной дроби в конечном числе мест, а также дописывание сверху или вычеркивание 
конечного числа звенъев цепной дроби не делают сходящуюся цепную дробь расходящейся и наоборот. Назовем цепную дробь (11) сходящейся, если сходится последовательность проективных векторов $\left\{\overline{\mathbf{u}}_{n}\right\}_{n=0}^{\infty}$. Для представителей классов эквивалентности, отвечающих последовательности проективных векторов, можно также рассматривать определители вида (20). Последовательность проективных векторов, связанных с некоторой цепной дробью, от произвольной последовательности проективных векторов отличает неравенство нулю всех таких определителей (это свойство не зависит от выбора представителя).

Если поле $F$ локально компактно, то проективное пространство $F \mathbb{P}^{m}$ - компакт, и в нем всякая бесконечная последовательность имеет предельную точку. Любое замкнутое множество будет множеством предельных точек некоторой последовательности. Множество предельных точек последовательности подходящих дробей цепной дроби (соответственно произвольных проективных векторов) назовем множеством значений иепной дроби (соответственно последовательности). Это замкнутое множество. Сходимость цепной дроби (последовательности) означает, что множество ее значенийодна точка.

Если в поле $F$ нет изолированных точек (достаточно, чтобы 0 не был изолированной точкой), то для любой счетной последовательности точек пространства $F \mathbb{P}^{m}$ можно указать сколь угодно близкую к ней последовательность, для которой составленные из ее координат определители матриц $\Delta_{n, 0}$ отличны от нуля. Вторую последовательность, как мы видели, можно рассматривать как последовательность знаменателей и числителей подходящих дробей некоторой цепной дроби. Поэтому любое множество предельных точек второй, а вместе с ней и первой последовательности будет множеством значений некоторой цепной дроби. Справедлива

ТЕорема 4. Если поле $F$ - сепарабельное метрическое пространство без изолированных точек, то сепарабельным будет и $F \mathbb{P}^{m}$, и любое его замкнутое подмножество служит множеством значений некоторой иепной дроби.

6. Обобщение теоремы Пинкерле. Изучим детальнее структуру конечномерных подпространств пространства $F^{\infty}$. Зафиксируем такое пространство $\mathscr{S}$ размерности $m+1$. Его подпространство $\mathscr{I}_{1}$ назовем относительно минимальным, если для каждой последовательности $\left\{h_{n}\right\}$ из $\mathscr{S}_{1}$ и любой последовательности $\left\{g_{n}\right\}$ из дополнения $\mathscr{S} \backslash \mathscr{S}_{1}$ существует и равен нулю предел отношения

$$
\lim _{n \rightarrow \infty} \frac{h_{n}}{g_{n}}=0 .
$$

Из двух относительно минимальных пространств $\mathscr{S}_{1}$ и $\mathscr{S}_{2}$ одно содержится в другом (при $\left\{h_{n}\right\} \in \mathscr{S}_{1} \backslash \mathscr{S}_{2},\left\{g_{n}\right\} \in \mathscr{S}_{2} \backslash \mathscr{S}_{1}$, получаем противоречие: $1=\lim _{n \rightarrow \infty}\left(h_{n} / g_{n}\right) \times$ $\left.\left(g_{n} / h_{n}\right)=0\right)$, т.е. относительно минимальные пространства вложены друг в друга. Всякое подпространство конечномерного пространства конечномерно. $k$-мерное относительно минимальное подпространство пространства $\mathscr{S}$ обозначим $\mathscr{S}^{(k)}$. Его элементы назовем $k$-минимальными последовательностями, а при $k=1$ - просто минимальными последовательностями. Последовательности-решения системы рекуррентных уравнений $\mathscr{R}$ - назовем минимальными ( $k$-минимальными) решениями системы $\mathscr{R}$. Базис в меньшем относительно минимальном пространстве может быть дополнен до базиса в пространстве большей размерности. Мы будем этим пользоваться. 
Когда и какие минимальные пространства существуют, можно увидеть, изучая $n p e-$ дельно периодические иепные дроби в $F=\mathbb{C}$. По определению их рекуррентные соотношения (18) имеют пределы по подпоследовательностям индексов, кратньм $T \in \mathbb{N}$ :

$$
\lim _{n \rightarrow \infty} p_{j, n T+k}=\widetilde{p}_{j, k} \in \mathbb{C}, \quad j=1, \ldots, m+1, \quad k=1, \ldots, T, \quad \widetilde{p}_{1, k} \neq 0 .
$$

Произведению $T$ предельных матриц $\widetilde{P}_{k}$ вида (13) отвечает характеристическое уравнение $\operatorname{det}\left(\widetilde{P}_{1} \cdots \widetilde{P}_{T}-\lambda E\right)=0$. Это уравнение степени $m+1$ по $\lambda$ с ненулевым свободным членом $\pm \widetilde{p}_{1,1} \cdots \widetilde{p}_{1, T}$. При $T=1$ (или $m=1$ ) картина такова. Занумеруем корни характеристического уравнения в порядке убывания модулей: $\left|\lambda_{1}\right| \geqslant \cdots \geqslant\left|\lambda_{m+1}\right|>0$. Если модули всех $\lambda$ различны, то по теореме Пуанкаре (см. $[11$, гл. $5, \S 5])$ сушествует решение $\mathbf{t}_{l}$ системы рекуррентных уравнений, для которого предел отношения $\left(\mathbf{t}_{l}\right)_{n+1} /\left(\mathbf{t}_{l}\right)_{n}$ равен $\lambda_{l}$, и определены все пространства $\mathscr{S}^{(l)}=\left\langle\mathbf{t}_{1}, \ldots, \mathbf{t}_{l}\right\rangle$. В [12], [13] показано, что при меньшем ограничении $\left|\lambda_{1}\right|>\left|\lambda_{2}\right| \geqslant \cdots \geqslant\left|\lambda_{m+1}\right|>0$ существует пространство $\mathscr{S}^{(m)}$ (цепная дробь сходится). Там же установлено, что при $T>1, m>1$ картина может быть иной. Решение, асимптотика которого в смысле корня степени $n$ равна $\lambda_{1}$ на какой-то из $T$ подпоследовательностей индексов $s_{k}=\{n T+k\}_{n=0}^{\infty}$, на других подпоследовательностях индексов может иметь асимптотику, определяющуюся другим корнем $\lambda_{l}, l>1$, или даже равняться нулю. В общем случае причины, почему нет пределов $\lim _{n \rightarrow \infty} h_{n} / g_{n}$, могут быть различны: это и полная иррегулярность поведения компонент векторов, и похожая асимптотика $\left(\left(\mathbf{t}_{l}\right)_{n}=\lambda_{l}^{n}, l=1, \ldots, m+1\right.$, где $\lambda_{l}$ - различные числа, модули которых равны), и даже их периодичность $\left(\left(\mathbf{t}_{l}\right)_{n}=\delta_{l, 1+n}(\bmod m+1)\right)$.

Изучим связь сходимости и существования относительно минимальных подпространств. Относительно минимальные пространства определяются своими базисами. Переходя от относительно минимального пространства некоторой размерности $k+1$ к пространству размерности $k$, мы будем менять только $k$ элементов базиса $\mathscr{S}^{(k+1)} \subset \mathscr{S}$, оставляя неизменными $m+1-k$ остальньх элементов базиса пространства $\mathscr{S}$. Начнем с некоторого базиса $q_{1}^{(m+1)}, \ldots, q_{m+1}^{(m+1)}$ пространства $\mathscr{S}$ :

$$
q_{j, n}^{(m+1)} \stackrel{\text { def }}{=} q_{j, n}, \quad j=1, \ldots, m+1, \quad n=-m-1,-m, \ldots
$$

Сформируем из компонент этих последовательностей проективныевекторы $\overline{\mathbf{u}}_{n}=\left(\mathbf{q}_{1, n}\right.$ : $\left.\cdots: \mathbf{q}_{m+1, n}\right)$.

Сходимость последовательности $\left\{\overline{\mathbf{u}}_{n}\right\}$ в $F \mathbb{P}^{m}$ означает сходимость в одной из карт $F \mathbb{P}_{i_{m}}^{m}$, т.е. существование $m$ пределов $r_{j}^{(m)} \in F$ :

$$
r_{j}^{(m) \stackrel{\text { def }}{=}} \lim _{n \rightarrow \infty} \frac{q_{j, n}^{(m+1)}}{q_{i_{m}, n}^{(m+1)}}, \quad j \neq i_{m} .
$$

Определим новьй базис в $\mathscr{S}$, вычтя из элементов исходного базиса проекиию $\left\{r_{j}^{(m)} \times\right.$ $\left.q_{i_{m}, n}^{(m+1)}\right\}$ на них последовательности $\left\{q_{i_{m}, n}^{(m+1)}\right\}$. (Эта операция - действительно проек$m o p$, т.е. повторное ее применение не меняет результата.) Итак, включим в новый базис последовательность $\left\{q_{i_{m}, n}\right\}_{n=1}^{\infty}$, а вместо последовательностей $\left\{q_{j, n}\right\}_{n=1}^{\infty}, j \neq i_{m}$, возьмем $m$ линейно выражающихся через них последовательностей $\mathbf{q}_{j}^{(m)}$ :

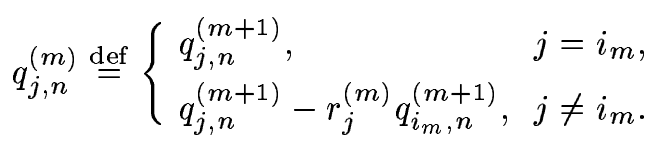


Последовательности $\mathbf{q}^{(m)}$ выражаются через последовательности $\mathbf{q}^{(m+1)}$ :

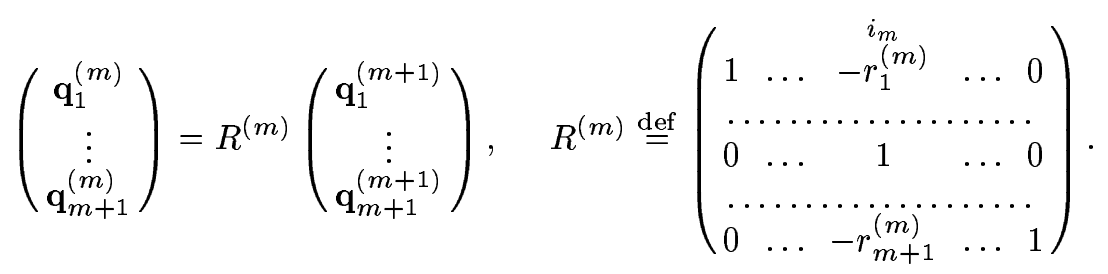

Натянутое на последовательности $\mathbf{q}_{j}^{(m)} m$-мерное пространство $\mathscr{S}$ будет относительно минимальным пространством. Действительно, равны нулю пределы

$$
\lim _{n \rightarrow \infty} \frac{q_{j, n}^{(m)}}{q_{i_{m}, n}}=\lim _{n \rightarrow \infty} \frac{q_{j, n}-r_{j}^{(m)} q_{i_{m}, n}}{q_{i_{m}, n}}=0
$$

Осталось заметить, что для того, чтобы установить относительную минимальность пространства $\mathscr{S}^{(k)}$, достаточно установить равенство нулю пределов $\lim _{n \rightarrow \infty} h_{n} / g_{n}$, где числителями берутся $k$ элементов его базиса, а знаменателями - остальные $m+1-k$ элементов базиса пространства $\mathscr{S}$.

Обратно, пусть теперь для $(m+1)$-мерного подпространства пространства $F^{\infty}$ существует $m$-мерное относительно минимальное пространство $\mathscr{S}^{(m)}$, последовательности $\mathbf{g}_{1}, \ldots, \mathbf{g}_{m}, \mathbf{g}_{i}=\left\{g_{i, n}\right\}_{n=-m-1}^{\infty}$, составляют его базис, а $\mathbf{g}_{m+1}-$ какой-то элемент, не содержащийся в $\mathscr{S}^{(m)}$. Составим из компонент векторов $\mathbf{g}_{i}$ проективные векторы $\mathbf{v}_{n}=\left(g_{1, n}: \cdots: g_{m+1, n}\right)$. Так как все отношения $g_{j, n} / g_{m+1, n}$ при $n \rightarrow \infty$ стремятся к 0 , то проективные векторы $\mathbf{v}_{n}$ сходятся к проективному вектору $\mathbf{e}_{m+1}=(0: \cdots: 0: 1)$. Для любого другого базиса $\mathbf{h}_{1}, \ldots, \mathbf{h}_{m+1}$,

$$
h_{i, n}=\sum_{j=1}^{m+1} c_{i, j} g_{j, n}, \quad \operatorname{det}\left(c_{i, j}\right) \neq 0
$$

и для тех $k$, для которых $c_{k, m+1} \neq 0$, также существуют пределы

$$
\lim _{n \rightarrow \infty} \frac{h_{i, n}}{h_{k, n}}=\lim _{n \rightarrow \infty} \frac{\sum_{j=1}^{m+1} c_{i, j} g_{j, n}}{\sum_{j=1}^{m+1} c_{k, j} g_{j, n}}=\lim _{n \rightarrow \infty} \frac{\sum_{j=1}^{m+1} c_{i, j} g_{j, n} / g_{m+1, n}}{\sum_{j=1}^{m+1} c_{k, j} g_{j, n} / g_{m+1, n}}=\frac{c_{i, m+1}}{c_{k, m+1}}
$$

Таким образом, проективные векторы сходятся в карте $F \mathbb{P}_{k}^{m}$.

ТеоремА 5. Последовательность тогда и только тогда, когда у отвечающего ей конечномерного подпространства пространства $F^{\infty}$ существует странство.

Следствием теоремы 5 будет следующее утверждение. 
ТЕоремА 6. т-мерная иепная дробь сходится тогда и только тогда, когда в подпространстве $\mathscr{S}(\mathscr{R})$ существует т-мерное относительно минимальное подпространство.

В случае $m=1$ теорема 6 преврашается в первую часть приведенной во введении теоремы Пинкерле [2], [3].

Теорему 5 можно применить к любому относительно минимальному пространству $\mathscr{S}^{(k+1)}$ пространства $\mathscr{S}$. Имея базис $\left(\mathbf{q}_{1}^{(k+1)}, \ldots, \mathbf{q}_{k+1}^{(k+1)}\right)$ пространства $\mathscr{S}^{(k+1)}$, можно рассмотреть последовательность $k$-мерных проективных векторов с компонентами, составленными из $n$-х членов $k+1$ базисной последовательности $\mathbf{q}_{i}^{(k+1)}$. Ее сходимость в $F \mathbb{P}^{k}$ эквивалентна существованию относительно минимального пространства $\mathscr{S}^{(k)}$. Поэтому справедлив следующий результат.

ТЕОрема 7. Если в $\mathscr{S}$ найдутся относительно минимальные пространства всех размерностей $m, m-1, \ldots, k$, то справедливы следующие формуль, задающие базис $\mathbf{q}_{1}^{(k)}, \ldots, \mathbf{q}_{k}^{(k)}$ пространства $\mathscr{S}^{(k)}$ :

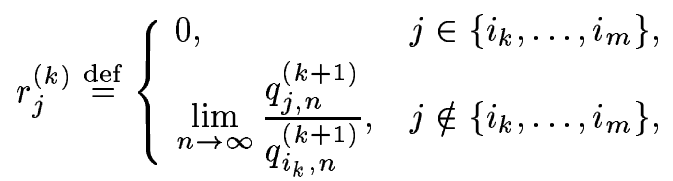

$$
\begin{aligned}
& q_{j, n}^{(k)} \stackrel{\text { def }}{=} q_{j, n}^{(k+1)}-r_{j}^{(k)} q_{i_{k}, n}^{(k+1)},
\end{aligned}
$$

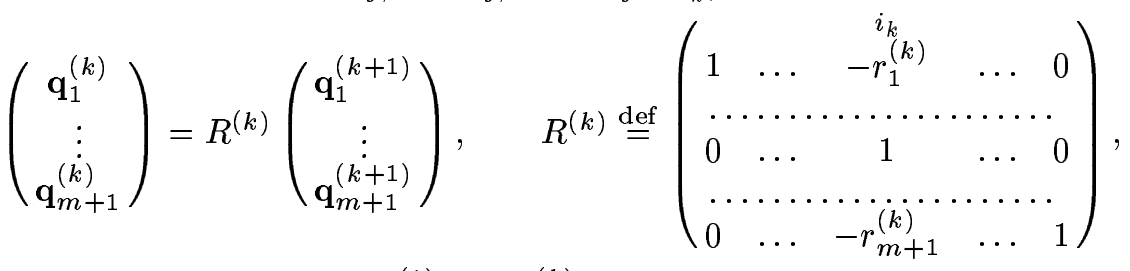

$$
\begin{aligned}
& \mathscr{S}^{(k)}=\left\langle\mathbf{q}_{i_{1}}^{(1)}, \ldots, \mathbf{q}_{i_{k}}^{(k)}\right\rangle, \quad k=1, \ldots, m .
\end{aligned}
$$

Часть Б) теоремы Пинкерле дает явньй вид гарантированных теоремой 3 двучленных рекуррентных соотношений, которым удовлетворяют минимальные решения. Перейдем теперь к доказательству утверж дения, обобщающего это свойство.

Выпишем выражение проективного вектора $\overline{\mathbf{f}}_{0}$ через компоненты $\overline{\mathbf{f}}_{n+1}$ :

$$
\overline{\mathbf{f}}_{0}=\mathscr{J}^{-1} Q_{n} \mathscr{J} \overline{\mathbf{f}}_{n+1}=\left(s_{2}: \cdots: s_{m+1}: s_{1}\right),
$$

где суммы $s_{j}$ равны

$$
q_{j, n-m} f_{m+1, n+1}+\sum_{k=1}^{m} q_{j, n-m+k} f_{k, n+1} .
$$

Пусть предел $\overline{\mathbf{f}}_{0}$ сходящейся цепной дроби лежит в $l$-й карте $(l \in\{1, \ldots, m+1\})$, координаты в которой задаются отображением $\sigma_{l}$ :

$$
\sigma_{l}\left(f_{1}, \ldots, f_{m}\right) \stackrel{\text { def }}{=}\left(f_{1}: \cdots: 1: \cdots: f_{m}\right) .
$$

Итак, пусть

$$
\sigma_{l}^{-1} \overline{\mathbf{f}}_{0}=\sigma_{l}^{-1}\left(f_{1,0}^{(l)}: \cdots: \stackrel{l}{1}: \cdots: f_{m+1,0}^{(l)}\right)=\left(f_{1,0}^{(l)}, \ldots, f_{l-1,0}^{(l)}, f_{l+1,0}^{(l)}, \ldots, f_{m+1,0}^{(l)}\right) .
$$


Равенство проективных векторов можно записать в виде равенства их прообразов в $l$-й карте, домножив которые на неравный нулю знаменатель

$$
q_{l, n-m} f_{m+1, n+1}+\sum_{k=1}^{m} q_{l, n-m+k} f_{k, n+1}
$$

компонент вектора $\sigma_{l}^{-1} \mathscr{J}^{-1} Q_{n} \mathscr{J} \overline{\mathbf{f}}_{n+1}$, можно переписать как $m$ линейных равенств. Предварительно вводя обозначение

$$
q_{j, r}^{\prime}= \begin{cases}q_{j, r}-f_{j, 0}^{(l)} q_{l, r}, & j<l \\ q_{j+1, r}-f_{j+1,0}^{(l)} q_{l, r}, & j>l\end{cases}
$$

выпишем их:

$$
f_{m+1, n+1} q_{j, n-m}^{\prime}+\sum_{k=1}^{m} f_{k, n+1} q_{j, n-m+k}^{\prime}=0, \quad j=1, \ldots, m .
$$

Если $f_{m+1, n+1} \neq 0$, то последние равенства переписьваются в виде соотношений

$$
q_{l, n}^{\prime}=\sum_{k=1}^{m-1} \frac{-f_{k, n+1}}{f_{m+1, n+1}} q_{l, n-m+k}^{\prime}+\frac{-f_{m, n+1}}{f_{m+1, n+1}} q_{l, n-m}^{\prime} .
$$

Если же и $f_{m, n+1} \neq 0$, то $(23)$ будут $(m+1)$-членными рекуррентными соотношениями, отвечающими цепной дроби

$$
\mathbf{b}_{0}^{\prime}+\frac{a_{0}^{\prime}}{\ddots \cdot+\frac{a_{n-1}^{\prime}}{\mathbf{b}_{n}^{\prime}+\frac{a_{n}^{\prime}}{\ddots}}}
$$

с элементами

$$
a_{n}^{\prime}=\frac{-f_{m+1, n+1}}{f_{m, n+1}} \in F^{\prime}, \quad \mathbf{b}_{n}^{\prime}=\left(\frac{f_{1, n+1}}{f_{m+1, n+1}}, \ldots, \frac{f_{m-1, n+1}}{f_{m+1, n+1}}\right) \in F^{m}
$$

Отметим, что компоненты проективных векторов $\overline{\mathbf{f}}_{n+1}$ вошли в коэффициенты (23) в виде вполне определенных отношений. Соотношения (23) можно применять для численного построения базиса в пространстве $m$-минимальных решений исходных рекуррентных соотношений.

Итак, доказана

ТЕОрема 8. Если в $\mathscr{S}$ есть относительно минимальное пространство максимальной размерности $\mathrm{m}$, то его базис составляют последовательности - числители и знаменатель подходящих дробей $\kappa(m-1)$-мерной чепной дроби (23).

Наша формулировка отличается от формулировки пункта Б) теоремы Пинкерле, поэтому посмотрим, что мы получили при $m=1$. Рекуррентным соотношениям, записанным в форме (3), отвечает цепная дробь с элементами $a_{n}=d_{n-1}^{-1}, b_{n}=d_{n-1}^{-1} c_{n-1}$. По 
доказанному нами минимальные решения $q_{n}^{\prime}$ подчинены условию

$$
\begin{aligned}
\frac{q_{n}^{\prime}}{q_{n-1}^{\prime}}=-\frac{1}{f_{1, n+1}} & =-\frac{1}{b_{n+1}+\frac{a_{n+1}}{b_{n+2}+\frac{a_{n+2}}{\ddots}}} \\
& =-\frac{1}{d_{n}^{-1} c_{n}+\frac{d_{n}^{-1}}{d_{n+1}^{-1} c_{n+1}+\frac{d_{n+1}^{-1}}{\ddots}}}=-\frac{d_{n}}{c_{n}+\frac{d_{n+1}}{c_{n+1}+\frac{d_{n+2}}{\ddots}}}
\end{aligned}
$$

что совпадает с утверждением Б) теоремы Пинкерле.

В заключение автор благодарит А. Д. Брюно за внимание к работе.

\section{СПИСОК ЦИТИРОВАННОЙ ЛИТЕРАТУРЫ}

[1] Gautschi W. Computational aspects of three-term recurrence relations // SIAM Review. 1967. V. 9. P. 24-82.

[2] Pincherle S. Delle funzioni ipergeometriche e di varie questioni ad esse attinenti // Giorn. Mat. Battaglini. 1894. V. 32. P. 209-291.

[3] Джоунс У., Трон В. Непрерывные дроби. Аналитическая теория и приложения. М.: Мир, 1985.

[4] Парусников В. И. Обобщение теоремы Пинкерле для $k$-членных рекуррентных соотношений // Препринт № 87. М.: ИПМ им. М.В. Келдыша РАН, 2000.

[5] Jacobi C. G. J. Allgemeine Theorie der kettenbruchänlichen Algorithmen, in welchen jede Zahl aus drei vorhergehenden gebildet wird // J. Reine Angew. Math. 1868. V. 69. P. 29-64.

[6] Euler L. De relatione inter ternas pluresve quantitates instituenda // Petersburger Akademie Notiz: Exhib. August 14, 1775.

[7] Perron O. Grundlagen für eine Theorie des Jacobischen Kettenbruchalgorithmus // Math. Ann. 1907. V. 64. №6. P. 1-76.

[8] Paley R. E. A. C., Ursell H. D. Continued fractions in several dimensions // Proc. Cambridge Phil. Soc. 1930. V. 26. № 2. P. 127-144.

[9] Парусников В. И. Алгоритм Якоби-Перрона и совместное приближение функций // Матем. сб. 1981. Т. 114 (156). № 2. С. 322-333.

[10] de Bruijn M. G. The interruption phenomenon for generalized continued fractions // Bull. Austral. Math. Soc. 1978. V. 19. № 2. P. 245-272.

[11] Гельфонд А. О. Исчисление конечных разностей. 3-е изд. М.: Наука, 1967.

[12] Парусников В. И. Предельно периодические многомерные непрерьвные дроби // Препринт № 62. М.: ИПМ им. М. В. Келдыша РАН, 1983.

[13] Parusnikov V. I. On the convergence of the multidimensional limit-periodic continued fractions // Lect. Notes in Math. 1985. № 1237. P. 217-227.

Институт прикладной математики им. М. В. Келдьша РАН

Поступило

E-mail: parus@spp.keldysh.ru

11.01.2003

Исправленный вариант

26.11.2004 